\title{
Atrial high rate episodes in patients with cardiac implantable electronic devices: implications for clinical outcomes
}

\author{
Kazuo Miyazawa ${ }^{1}$. Daniele Pastori ${ }^{2}$ - Yan-Guang $\mathrm{Li}^{1,3}$. Orsolya Székely ${ }^{1} \cdot$ Farhan Shahid $^{1}$. Giuseppe Boriani ${ }^{4}$. \\ Gregory Y. H. Lip ${ }^{1,5,6}$
}

Received: 9 November 2018 / Accepted: 4 February 2019 / Published online: 13 February 2019

(c) The Author(s) 2019

\begin{abstract}
Background Atrial high rate episodes (AHREs) detected by cardiac implantable electronic devices (CIEDs) are associated with an increased risk of stroke. However, the impact of AHRE on improving stroke risk stratification scheme remains uncertain.

Objective The purpose of this study was to assess the impact of AHRE on prognosis in relation with cardiovascular events and risk stratification.

Methods A total of 856 consecutive patients who had dual-chamber CIEDs implanted were retrospectively analyzed. To detect AHREs, they were monitored for 6 months after CIEDs' implantation and were followed for a mean of 4.0 years for clinical outcomes such as thromboembolism or death.

Results Overall, 125 (14.6\%) of patients developed AHREs within the first 6 months (median age 72.0 years, 39.3\% female). Patients with AHREs had a high rate of thromboembolism (2.6\%/year) and mortality (3.0\%/year). On multivariate analysis, AHRE was significantly associated with increased risk of thromboembolism [hazard ratio (HR) 3.40; 95\% confidence interval (CI) 1.38-8.37, $P=0.01]$ and death (HR 3.47; 95\% CI 1.51-7.95; $P<0.01$ ). The predictive abilities of the $\mathrm{CHADS}_{2}$ and $\mathrm{CHA}_{2} \mathrm{DS}_{2}$-VASc scores were modest, with no significant improvements by adding AHRE to those scores. However, the integrated discrimination improvement and net reclassification improvement showed that the addition of AHRE to the $\mathrm{CHADS}_{2}$ and $\mathrm{CHA}_{2} \mathrm{DS}_{2}$-VASc scores statistically improved their predictive ability for the composite outcome.

Conclusions AHRE was an independent factor associated with increased risk of clinical outcomes. The addition of AHRE to the clinical risk scores significantly improved discrimination for thromboembolism or death.
\end{abstract}

Keywords Atrial fibrillation $\cdot$ Atrial high rate episode $\cdot$ Cardiac implanted electronic device $\cdot$ Risk stratification scheme

\section{Introduction}

Electronic supplementary material The online version of this article (https://doi.org/10.1007/s00392-019-01432-y) contains supplementary material, which is available to authorized users.

\footnotetext{
Gregory Y. H. Lip

gregory.lip@liverpool.ac.uk

1 Institute of Cardiovascular Sciences, University of Birmingham, Birmingham, UK

2 Department of Internal Medicine and Medical Specialties, I Clinica Medica, Atherothrombosis Center, Sapienza University of Rome, Rome, Italy

3 Department of Cardiology, Chinese PLA Medical School, Chinese PLA General Hospital, Beijing, China
}

Atrial fibrillation (AF) is the most common sustained arrhythmia, and is associated with increased risks of morbidity and mortality [1]. Integrated management including anticoagulation therapy in patients with AF is increasingly

4 Cardiology Division, Department of Biomedical, Metabolic and Neural Sciences, University of Modena and Reggio Emilia, Policlinico di Modena, Modena, Italy

5 Liverpool Centre for Cardiovascular Science, University of Liverpool and Liverpool Heart and Chest Hospital, Liverpool, UK

6 Thrombosis Research Unit, Department of Clinical Medicine, Aalborg University, Aalborg, Denmark 
recognized [2, 3], but there are still a substantial number of AF patients admitted with stroke, heart failure, and other complications $[4,5]$. This can be to some extent due to asymptomatic nature of AF as approximately one-third of patients did not report any symptoms commonly attributable to AF (e.g., palpitations, shortness of breath, or chest pain) [6], leading to a prolonged delay in AF diagnosis and timely initiation of anticoagulation therapy. In many cases, asymptomatic AF is diagnosed only after the onset of complications such as ischemic stroke or congestive heart failure has occurred [7].

Subclinical and asymptomatic atrial tachyarrhythmias often precede the development of clinical AF, and can be detected by continuous cardiac monitoring technology including cardiac implantable electronic devices (CIEDs, e.g., permanent pacemakers, implantable cardioverter defibrillators [ICD], and cardiac resynchronization therapy [CRT] devices) [8]. Previous studies demonstrated that atrial high rate episodes (AHREs) detected by CIEDs have a high correlation with clinically documented AF [9], and are independently associated with an increased risk of ischemic stroke and systemic embolism [10-12].

Various efforts have been made to identify the risk factors for the development of AHRE and to assess the relationship between AHREs and clinical outcomes in patients with CIEDs. Although recent studies have investigated the impact of AHRE on the management of patient with AHREs, the optimal management of such patients remains uncertain in the current clinical guidelines, especially anticoagulation therapy in patients with AHREs [13].

In this study, we aimed to investigate the clinical characteristics of AHRE in a 'real-world' cohort of patients with CIEDs, and assessed the impact of AHRE on prognosis in relation with cardiovascular events and clinical event-risk stratification schemes.

\section{Methods}

\section{Study population}

The consecutive patients receiving pacemaker, ICD, and CRT devices, who attended the cardiology department of Sandwell and West Birmingham Hospitals NHS Trust (Sandwell General Hospital and City Hospital) in Birmingham, United Kingdom were retrospectively enrolled. In the present study, patients with single-chamber CIEDs (i.e., VVI and AAI devices) and patients with $<6$ months of follow-up were excluded.

We retrospectively reviewed the patients' medical records, and collected clinical information on demographics, co-morbidities, and concomitant medications. Prior history of AF was defined as a documented AF on 12-lead ECG or Holter ECG monitoring.

\section{Atrial high rate episode and clinical outcomes}

The device diagnostic information was interrogated to assess whether patients had developed AHREs or not within the first 6 months since the time of CIEDs' implantation. All CIEDs were programmed to the nominal setting, which detected any episodes of arrhythmia. We defined the AHRE as an episode lasting at least 5 min with atrial rate $\geq 175$ beats per minute, given that previously published studies suggested that the 5 min cut-off value excluded most episodes of over-sensing due to mechanical problems and appropriately detected clinical AF [9]. Device diagnostic information on AHREs was reviewed by at least 1 experienced electrophysiologist, blinded to clinical outcomes.

The endpoint for the present study was the occurrence of thromboembolism (ischemic stroke, transient ischemic attack [TIA], or systemic embolism) or all-cause death 6 months after CIEDs' implantation. Baseline characteristics of patients with and without AHREs were compared, and the predictive ability of $\mathrm{CHADS}_{2}$ and $\mathrm{CHA}_{2} \mathrm{DS}_{2}-\mathrm{VASc}$ scores for clinical outcomes was assessed. This study was conducted in accordance with the EU Guidance on Good Clinical Practice CPMP/ ECH/135/95. The present study was approved by the local research ethics committee and complied with the Declaration of Helsinki. All patients included in the study had there data anonymised.

\section{Statistical analysis}

Continuous variables were presented as means and standard deviations (SD), unless not normally distributed, in which case medians and interquartile ranges (IQR) were used. Categorical variables were presented as frequencies and percentages. Censoring was done for the first event recorded. We compared categorical variables using Chi-square test and continuous variables using independent samples $t$ test for normally distributed data or Mann-Whitney $U$ test for nonnormal distribution. Baseline characteristics, stroke risk profiles, and medications were tabulated between patients with and without AHREs. Annual incidence rates for the composite and individual endpoints were recorded in patients with and without AHREs. Kaplan-Meier survival curves were depicted, and differences were assessed by log-rank test. The independent effects of AHRE on the clinical outcomes were assessed using a Cox proportional hazards regression model including components of the $\mathrm{CHA}_{2} \mathrm{DS}_{2}$-VASc score (age assessed as a continuous variable), prior history of AF, and oral anticoagulant (OAC) use as co-varieties.

Receiver-operating characteristic (ROC) curve analysis was performed to test the predictive discrimination of the 
risk scores for clinical outcomes based on an area under the ROC curve (AUC). To compare the predictive ability of the predictive models, we calculated the statistical difference between the AUCs with the method of DeLong et al. [14] Furthermore, improvements in the predictive accuracy of the models were evaluated by calculating the integrated discrimination improvement (IDI) and the net reclassification improvement (NRI), as described by Pencina et al. [15].

We also assessed the clinical usefulness and net benefit of the predictive models using decision curve analysis (DCA), as described by Vickers et al. [16]. This analysis identifies patients who will have any of the adverse events evaluated, based on the predictions of the modified risk score in comparison with the original. The clinical net benefit is calculated by summing the benefits (true positive) and subtracting the harms (false positive). The result of this analysis is presented with the selected probability threshold plotted on the $x$-axis and the benefit of the evaluated model on the $y$-axis.

All statistical analyses were performed using IBM SPSS Statistics version 24.0 software (IBM Corp) and R software packages version 3.5.1 (R Development Core Team).
Statistical significance was set as a two-sided $P$ value of $<0.05$.

\section{Results}

\section{Baseline characteristics}

Baseline characteristics of patients with and without AHREs are shown in Table 1. Median age of the patients was 72.0 (IQR: 62.0-80.0) years, and 336 (39.3\%) were female. Of 856 patients with CIEDs, $74.6 \%$ had pacemaker, $15.0 \%$ ICD, and $10.4 \%$ CRT. During a mean follow-up of $48.2 \pm 32.3$ months, 125 (14.6\%) of patients developed AHREs in the first 6 months. Patients with AHREs were older, with a higher prevalence of prior AF (and accordingly higher use of oral anticoagulants and digoxin, lower use of antiplatelets), compared to those without AHREs. No significant differences in mean $\mathrm{CHADS}_{2}$ and $\mathrm{CHA}_{2} \mathrm{DS}_{2}$-VASc scores were found between two groups.
Table 1 Baseline characteristics of patients with and without AHRE

\begin{tabular}{|c|c|c|c|c|}
\hline & Overall $(n=856)$ & $\operatorname{AHRE}(n=125)$ & No AHRE $(n=731)$ & $P$ value \\
\hline \multicolumn{5}{|l|}{ Demographics } \\
\hline Age, median (IQR) & $72.0(62.0-80.0)$ & $74(63.0-81.0)$ & $71.0(62.0-79.0)$ & 0.03 \\
\hline Age $65-74$ y & $232(27.1)$ & $29(23.2)$ & $203(27.8)$ & 0.29 \\
\hline Age $>75$ y & $383(44.7)$ & $66(52.8)$ & $317(43.4)$ & 0.05 \\
\hline Female gender [no., $(\%)]$ & $336(39.3)$ & $342(33.6)$ & $294(40.2)$ & 0.16 \\
\hline BMI, median (IQR) & $27.9(24.6-31.7)$ & $27.4(23.5-31.8)$ & $28.0(24.6-31.5)$ & 0.41 \\
\hline \multicolumn{5}{|l|}{ Medical history [no., (\%)] } \\
\hline Hypertension & $603(70.4)$ & $91(72.8)$ & $512(70.0)$ & 0.53 \\
\hline Diabetes mellitus & $241(28.2)$ & $36(28.8)$ & $205(28.0)$ & 0.86 \\
\hline Dyslipidemia & $554(68.6)$ & $81(68.6)$ & $473(68.7)$ & 1.00 \\
\hline Heart failure & $214(25.0)$ & $39(31.2)$ & $175(23.9)$ & 0.08 \\
\hline Prior stroke/TIA & $92(10.7)$ & $15(12.0)$ & $77(10.5)$ & 0.63 \\
\hline Vascular disease & $317(37.0)$ & $48(38.4)$ & $269(36.8)$ & 0.73 \\
\hline Prior history of AF & $212(24.8)$ & $75(60.0)$ & $137(18.7)$ & $<0.001$ \\
\hline \multicolumn{5}{|c|}{ Thromboembolic risk, mean \pm SD } \\
\hline $\mathrm{CHADS}_{2}$ score & $1.9 \pm 1.2$ & $2.0 \pm 1.3$ & $1.9 \pm 1.2$ & 0.07 \\
\hline $\mathrm{CHA}_{2} \mathrm{DS}_{2}$-VASc score & $3.4 \pm 1.6$ & $3.5 \pm 1.7$ & $3.3 \pm 1.6$ & 0.22 \\
\hline \multicolumn{5}{|l|}{ Medications [no., $(\%)]$} \\
\hline Beta-blocker & $270(35.5)$ & $47(40.9)$ & $223(34.5)$ & 0.19 \\
\hline ACE-I/ARB & $436(56.7)$ & $66(57.4)$ & $370(56.6)$ & 0.87 \\
\hline Diuretics & $276(35.8)$ & $42(36.5)$ & $234(35.7)$ & 0.87 \\
\hline Statin & $509(66.0)$ & $74(64.3)$ & $435(66.3)$ & 0.68 \\
\hline OAC & $151(19.7)$ & $54(47.0)$ & $97(14.9)$ & $<0.001$ \\
\hline Antiplatelet & $396(51.4)$ & $49(42.2)$ & $347(53.0)$ & 0.03 \\
\hline Digoxin & $38(4.9)$ & $12(10.4)$ & $26(4.0)$ & 0.003 \\
\hline
\end{tabular}

$A C E-I$ angiotensin-converting enzyme inhibitor, $A F$ atrial fibrillation, $A H R E$ atrial high rate episode, $A R B$ angiotensin II receptor blocker, $B M I$ body mass index, $I Q R$ interquartile range, $O A C$ oral anticoagulant, TIA transient ischemic attack 


\section{Clinical outcomes and atrial high rate episode}

During the follow-up, the observed rates of thromboembolism, all-cause death and composite outcome were $4.2 \%$ $(n=36), 5.4 \%(n=46)$ and $9.3 \%(n=80)$, respectively (Table 2). Patients with AHREs had a higher risk of thromboembolism (incidence 2.6\%/year) compared with those without AHREs (incidence 0.9\%/year) [hazard ratio (HR) 2.703, 95\% confidence interval (CI) 1.52-4.81, $P<0.001]$. Similarly, patients with AHREs had higher risk of all-cause death and composite outcome (thromboembolism or allcause death) than those without AHREs (3.0\%/year vs. $1.1 \%$ / year, HR 3.85, 95\% CI 1.90-7.80, $P<0.001$ for all-cause death; 5.4\%/year vs. 2.0\%/year, HR 3.32, 95\% CI 1.95-5.65, $P<0.001$ for the composite outcome). In a subgroup analysis of patients who had no prior history of AF, patients with AHREs tended to have a higher risk of thromboembolism compared with those without AHREs, but not statistically significant. On the other hand, patients with AHREs had a significantly higher risk of all-cause death compared with those without AHREs (Supplementary Table 1).

Kaplan-Meier curve analysis shows that crude event-free survival for thromboembolism, all-cause death and composite outcome appeared to be lower in patients with AHREs than those without AHREs $(P=0.006, P<0.001, P<0.001$, respectively) (Fig. 1). On multivariate Cox proportional hazards regression analysis (Table 3 ), age was significantly associated with increased risk of all-cause death and the composite outcome (HR 1.13, 95\% CI 1.08-1.18, $P<0.001$, HR 1.06, 95\% CI 1.03-1.09, $P<0.001$, respectively); heart failure with all-cause death (HR 2.21, 95\% CI 1.03-4.74, $P=0.04)$; prior stroke or TIA with thromboembolism and composite outcome (HR 2.64, 95\% CI 1.16-5.99, $P=0.02$, HR 2.23, 95\% CI 1.25-3.96, $P=0.006$, respectively). Prior history of AF was not an independent factor associated with any outcomes (all $P>0.05$ ). Similarly, the use of OAC was not significantly associated with any outcomes (all $P>0.05$ ), although the association trended towards being protective.

On multivariate adjustment, AHRE was significantly associated with increased risk of all outcomes (HR 3.40, 95\% CI 1.38-8.37, $P=0.008$ for thromboembolism, HR $3.47,95 \%$ CI $1.51-7.95, P=0.003$ for all-cause death, and
HR 3.52, 95\% CI 1.89-6.55, $P<0.001$ for the composite outcome).

When we performed a subgroup analysis of patients without prior history of $\mathrm{AF}(n=644)$ and those with prior history of AF $(n=212)$, multivariate Cox regression analysis showed that the impact of AHRE slightly weakened rather than entire population, but still significant on the clinical outcomes, especially all-cause death and the composite outcome (Supplementary Tables 2 and 3).

\section{Adding AHRE to the $\mathrm{CHADS}_{2}$ and $\mathrm{CHA}_{2} \mathrm{DS}_{2}$-VASC scores for predicting clinical outcomes}

ROC curve analysis showed that the predictive abilities of the $\mathrm{CHADS}_{2}$ and $\mathrm{CHA}_{2} \mathrm{DS}_{2}$-VASc scores were modest and there were no significant improvements after adding AHRE to the $\mathrm{CHADS}_{2}$ and $\mathrm{CHA}_{2} \mathrm{DS}_{2}$-VASc scores $\left(\mathrm{CHADS}_{2}\right.$ : AUCs 0.60 to $0.61, P=0.29$ for thromboembolism; AUCs 0.65 to $0.65, P=0.73$ for all-cause death; and AUCs 0.63 to $0.64, P=0.32$ for the composite outcome, $\mathrm{CHA}_{2} \mathrm{DS}_{2}$-VASc: AUCs 0.60 to $0.61, P=0.35$ for thromboembolism; AUCs 0.68 to $0.68, P=0.68$ for all-cause death; and AUCs 0.65 to $0.65, P=0.33$ for the composite outcome) (Table 4).

Based on the IDI and the NRI, the addition of AHRE to the $\mathrm{CHADS}_{2}$ and $\mathrm{CHA}_{2} \mathrm{DS}_{2}$-VASc scores statistically improved discriminative value for composite outcome $\left(\mathrm{CHADS}_{2}\right.$ : IDI $0.01, P=0.03$; NRI $0.20, P=0.04$, $\mathrm{CHA}_{2} \mathrm{DS}_{2}$-VASc: IDI $0.01, P=0.02$; NRI $0.20, P=0.04$ ), but not for thromboembolism and all-cause death (CHADS $_{2}$ : IDI $0.002, P=0.09$; NRI $0.23, P=0.12$, for thromboembolism, IDI $0.004, P=0.10$; NRI 0.20 , $P=0.13$, for all-cause death, $\mathrm{CHA}_{2} \mathrm{DS} 2-\mathrm{VASc}$ IDI 0.002 , $P=0.08$; NRI $0.22, P=0.18$, for thromboembolism; IDI $0.004, P=0.11$; NRI $0.20, P=0.13$, for all-cause mortality) (Table 4).

DCA graphically demonstrated that there were minimal net benefits of the addition of AHRE to the $\mathrm{CHADS}_{2}$ and $\mathrm{CHA}_{2} \mathrm{DS}_{2}$-VASc scores for predicting thromboembolism (Fig. 2a), all-cause death (Fig. 2b), and composite outcome (Fig. 2c).

Table 2 Clinical outcomes after first 6 months in patients with and without AHRE

\begin{tabular}{|c|c|c|c|c|c|c|c|}
\hline \multirow[t]{2}{*}{ Clinical outcomes } & \multirow[t]{2}{*}{$\begin{array}{l}\text { Overall }(n=856) \\
{[\text { no. }(\%)]}\end{array}$} & \multicolumn{2}{|c|}{$\begin{array}{l}\text { Patients with AHRE } \\
(n=125)\end{array}$} & \multicolumn{2}{|c|}{$\begin{array}{l}\text { Patients without AHRE } \\
(n=7310\end{array}$} & \multirow[t]{2}{*}{ Unadjusted HR (95\% CI) } & \multirow[t]{2}{*}{$P$ value } \\
\hline & & No. of events & $\% /$ year & No. of events & $\% /$ year & & \\
\hline Thromboembolism & $36(4.2)$ & 9 & 2.6 & 27 & 0.9 & $2.703(1.52-4.81)$ & $<0.001$ \\
\hline All-cause death & $46(5.4)$ & 11 & 3.0 & 35 & 1.1 & $3.845(1.90-7.80)$ & $<0.001$ \\
\hline
\end{tabular}

$A H R E$ atrial high rate episode, $C I$ confidence intervals, $H R$ hazard ratio 
Fig. 1 Kaplan-Meier curve analysis for thromboembolism (a), allcause death (b), and composite outcome (c)

\section{Discussion}

The main finding of the present study is that AHRE detected by CIEDs was an independent factor associated with significantly increased risks of thromboembolism and all-cause death, regardless of the presence or absence of prior history of AF. Second, the addition of AHREs to the $\mathrm{CHA}_{2} \mathrm{DS}_{2}$-VASc score statistically improved its discrimination ability for the composite outcome of thromboembolism or all-cause death. This is a contemporary 'real-world' cohort of patients with CIEDs, in relation with AHRE. To the best of our knowledge, no previous study has investigated the clinical impact of AHRE on risk stratification for thromboembolism and all-cause death.

The widespread use of the CIED technology in the management of a broad spectrum of cardiac diseases (i.e., bradycardia, life-threatening tachycardia, and heart failure) offers the long-term continuous ECG monitoring, which allows an early detection of atrial tachyarrhytmias including AF before they become clinically evident [17]. However, most of AHREs are asymptomatic, short-lasting, and hard to be detected by the conventional methods such as 12-lead ECG or ambulatory Holter ECG monitoring. The reported incidence of AHRE in patients with CIEDs is relatively variable across studies, ranging from 30 to $70 \%$ [18]. Although the incidence may strongly depend on the clinical profile of study population, the previous studies have consistently reported that AHRE is associated with a substantial risk of subsequent development of clinically diagnosed AF, and is also associated with an increased risk of ischemic stroke and death [10-12]. However, the absolute risk of stroke in patients with AHREs may be lower than in those with clinical AF.

In general, AF is known to be associated with fivefold risk of stroke compared to normal sinus rhythm, while recent meta-analysis showed that the annual rate of stroke in patients with AHREs was $1.89 / 100$ person-year with 2.4-fold increased risk of stroke compared to those without AHREs [19]. In the present study, AHRE was significantly associated with a threefold greater risk of thromboembolism and of all-cause death. Our findings reinforce the evidence that AHRE may not hold the same adverse prognosis as clinical AF, but may be considered as an early stage of clinical AF carrying an intermediate risk of stroke. On the other hand, the incidences of clinical outcomes in the present study were relatively higher than the previous studies including general AF population. This may be due to the differences in baseline medical history, as in our study, half of patients were elderly (i.e., $>75$ years) with a high proportion of hypertension, dyslipidemia, and vascular disease. The
A Thromboembolism

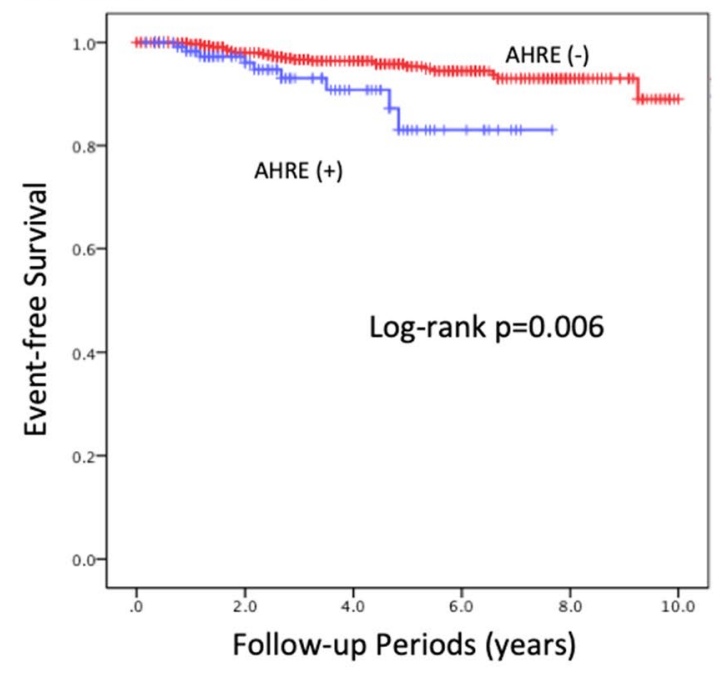

B All-cause death

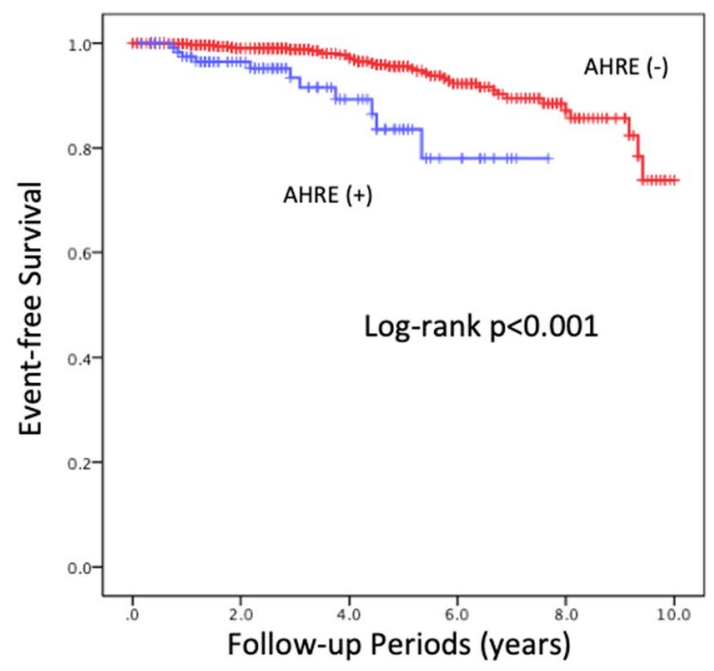

C Composite outcome

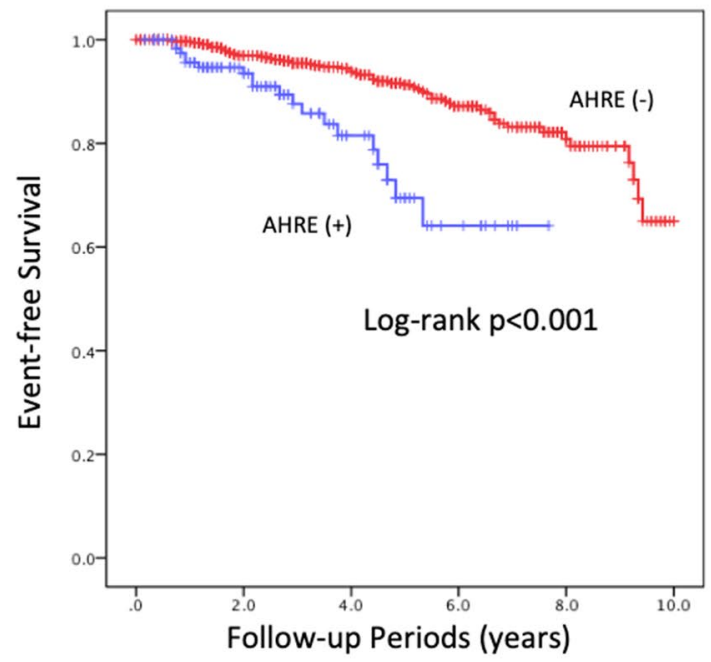


Table 3 Multivariable Cox regression analysis for clinical outcomes

\begin{tabular}{|c|c|c|c|c|c|c|}
\hline \multirow[t]{2}{*}{ Outcomes and variables } & \multicolumn{2}{|c|}{ Thromboembolism } & \multicolumn{2}{|l|}{ All-cause death } & \multicolumn{2}{|c|}{ Composite outcome } \\
\hline & HR $(95 \% \mathrm{CI})$ & $P$ value & HR $(95 \%$ CI $)$ & $P$ value & $\mathrm{HR}(95 \% \mathrm{CI})$ & $P$ value \\
\hline Age & $1.01(0.98-1.04)$ & 0.54 & $1.13(1.08-1.18)$ & $<0.001$ & $1.06(1.03-1.09)$ & $<0.001$ \\
\hline Female gender & $1.01(0.48-2.13)$ & 0.97 & $1.16(0.60-2.25)$ & 0.65 & $1.17(0.71-1.93)$ & 0.54 \\
\hline Hypertension & $1.28(0.50-3.26)$ & 0.61 & $0.80(0.36-1.79)$ & 0.59 & $0.97(0.53-1.77)$ & 0.91 \\
\hline Diabetes mellitus & $1.50(0.73-3.08)$ & 0.27 & $1.68(0.86-3.28)$ & 0.13 & $1.56(0.96-2.54)$ & 0.07 \\
\hline Heart failure & $0.60(0.24-1.53)$ & 0.29 & $2.20(1.03-4.74)$ & 0.04 & $1.31(0.73-2.35)$ & 0.37 \\
\hline Prior stroke/TIA & $2.64(1.16-5.99)$ & 0.02 & $2.17(0.99-4.73)$ & 0.05 & $2.23(1.25-3.96)$ & 0.01 \\
\hline Vascular disease & $1.64(0.78-3.42)$ & 0.19 & $1.03(0.50-2.10)$ & 0.94 & $1.28(0.77-2.14)$ & 0.34 \\
\hline Prior history of AF & $1.08(0.43-2.71)$ & 0.88 & $0.95(0.42-2.18)$ & 0.91 & $0.97(0.52-1.80)$ & 0.92 \\
\hline OAC use & $0.66(0.24-1.81)$ & 0.42 & $0.50(0.19-1.32)$ & 0.16 & $0.51(0.25-1.03)$ & 0.06 \\
\hline AHRE & $3.40(1.38-8.37)$ & 0.01 & $3.47(1.51-7.95)$ & 0.003 & $3.52(1.89-6.55)$ & $<0.001$ \\
\hline
\end{tabular}

Adjusted covariates including components of the $\mathrm{CHA}_{2} \mathrm{DS}_{2}$-VASc score (age assessed as a continuous variable), prior AF documentation, OAC use, and AHRE lasting at least $5 \mathrm{~min}$

$A F$ atrial fibrillation, $A H R E$ atrial high rate episode, $C I$ confidence intervals, $H R$ hazard ratio, $O A C$ oral anticoagulant, TIA transient ischemic attack

Table 4 Comparison of the ROC curve, IDI and NRI of the $\mathrm{CHADS}_{2}$ vs. $\mathrm{CHADS}_{2}+\mathrm{AHRE}$ and $\mathrm{CHA}_{2} \mathrm{DS}_{2}$-VASc vs. $\mathrm{CHA}_{2} \mathrm{DS}_{2}$-VASc + AHRE in predicting outcomes

\begin{tabular}{|c|c|c|c|c|c|c|c|}
\hline Clinical outcomes and risk scores & $\mathrm{C}$-statistic & $95 \% \mathrm{CI}$ & $P$ value* & IDI & $P$ value & NRI & $P$ value \\
\hline \multicolumn{8}{|l|}{ Thromboembolism } \\
\hline $\mathrm{CHADS}_{2}$ & 0.56 & $0.50-0.69$ & 0.29 & 0.002 & 0.09 & 0.23 & 0.12 \\
\hline $\mathrm{CHADS}_{2}+\mathrm{AHRE}$ & 0.61 & $0.52-0.70$ & & & & & \\
\hline $\mathrm{CHA}_{2} \mathrm{DS}_{2}$-VASc & 0.60 & $0.52-0.68$ & 0.35 & 0.002 & 0.08 & 0.22 & 0.18 \\
\hline $\mathrm{CHA}_{2} \mathrm{DS}_{2}-\mathrm{VASc}+\mathrm{AHRE}$ & 0.61 & $0.53-0.69$ & & & & & \\
\hline \multicolumn{8}{|l|}{ All-cause death } \\
\hline $\mathrm{CHADS}_{2}$ & 0.65 & $0.58-0.72$ & 0.73 & 0.004 & 0.100 & 0.20 & 0.13 \\
\hline $\mathrm{CHADS}_{2}+\mathrm{AHRE}$ & 0.65 & $0.58-0.72$ & & & & & \\
\hline $\mathrm{CHA}_{2} \mathrm{DS}_{2}$-VASc & 0.68 & $0.60-0.75$ & 0.68 & 0.004 & 0.11 & 0.20 & 0.13 \\
\hline $\mathrm{CHA}_{2} \mathrm{DS}_{2}-\mathrm{VASc}+\mathrm{AHRE}$ & 0.68 & $0.61-0.75$ & & & & & \\
\hline \multicolumn{8}{|l|}{ Composite outcome } \\
\hline $\mathrm{CHADS}_{2}$ & 0.63 & $0.57-0.69$ & 0.32 & 0.01 & 0.03 & 0.20 & 0.04 \\
\hline $\mathrm{CHADS}_{2}+\mathrm{AHRE}$ & 0.64 & $0.58-0.70$ & & & & & \\
\hline $\mathrm{CHA}_{2} \mathrm{DS}_{2}$-VASc & 0.65 & $0.59-0.70$ & 0.33 & 0.01 & 0.02 & 0.20 & 0.04 \\
\hline $\mathrm{CHA}_{2} \mathrm{DS}_{2}-\mathrm{VASc}+\mathrm{AHRE}$ & 0.65 & $0.60-0.71$ & & & & & \\
\hline
\end{tabular}

AHRE atrial high rate episode, $C I$ confidence interval, $I D I$ integrated discriminatory improvement, $N R I$ net reclassification improvement, $R O C$ receiver-operating characteristic

*For C-statistic comparison present study included patients with not only pacemaker, but also those with ICD and CRT, who frequently have left ventricular dysfunction and/or ischemic heart disease and are at risk of sudden cardiac death.

Interestingly, we found that prior history of clinical AF was more frequently observed in patients with AHREs, but this was not independent factor associated with any outcomes in Cox proportional hazards regression models. This can be explained by the fact that CIEDs' implantation itself could suppress new AF development. Previous studies have demonstrated that dual-chamber pacing modes reduce the incidence of AF in patients with sick sinus syndrome [20, 21]. In patients with CRT, hemodynamic improvement due to synchronized bi-ventricular pacing is reported to decrease the incidence of AF [22]. Furthermore, in a previous study of a heart failure cohort, there was no significant difference in the cumulative probability of developing thromboembolic events between AHRE patients with and without history of AF [23]. Of note, the subgroup analysis of patients without prior history of AF demonstrated that we found a significant association between AHRE and clinical outcomes, although there were a relatively small number of patients and clinical 

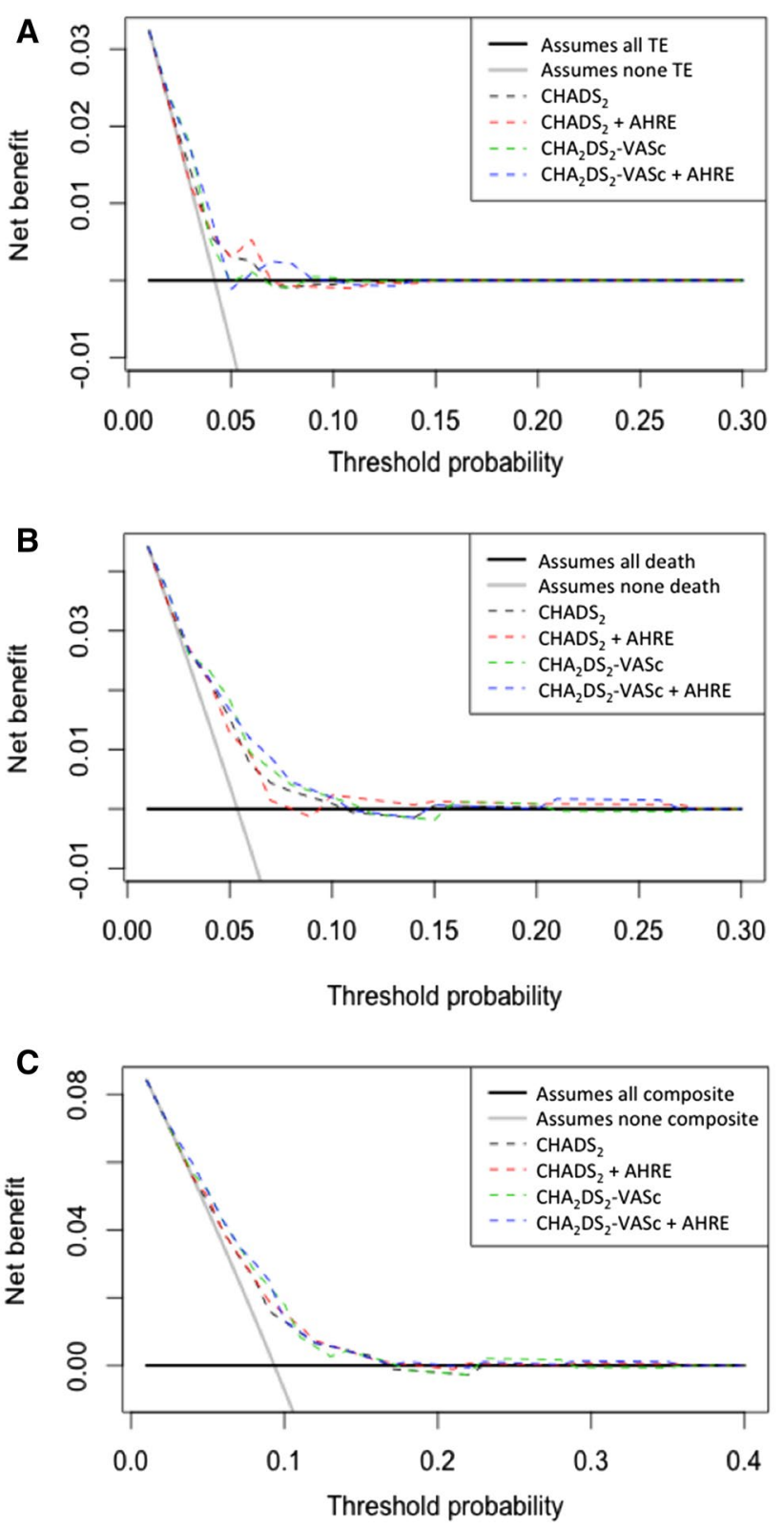

Fig. 2 Decision curve analysis for predicting cardiovascular events (a thromboembolism, $\mathbf{b}$ all-cause death, and $\mathbf{c}$ composite outcome)

events. Thus, current actual AF burden (i.e., AHRE) has a more significant impact on clinical outcomes rather than a previously documented history of AF.

In the present study, we showed the modest predictive abilities of the $\mathrm{CHADS}_{2}$ and the $\mathrm{CHA}_{2} \mathrm{D}_{2}$-VASc scores for risk of clinical outcomes, even in the subgroup of patients with and without prior history of AF. Possible explanations for the modest predictive performance in the CIED population include the differences in baseline medical history between the general AF population and CIEDs' population, and the suppression of new AF episodes after CIEDs' implantation, which may affect the predictive ability of clinical risk scores in this patient population. However, AHRE has previously been reported to help to refine eventrisk stratification in such patients. Botto et al. demonstrated that combination of the data on AHRE with the $\mathrm{CHADS}_{2}$ score divided patients with CIEDs into two subpopulations with significantly different risks of thromboembolic events [24]. Furthermore, Boriani et al. reported that the addition of AHRE burden to the $\mathrm{CHADS}_{2}$ and $\mathrm{CHA}_{2} \mathrm{DS}_{2}$-VASc scores significantly improved their C-statistics [25]. In the present study, ROC curve analysis indicated no significant improvements of $\mathrm{C}$-statistics by adding the data on AHREs to the clinical risk scores, but we showed a statistically significant improvement of its discriminatory value and a net clinical benefit using the IDI, NRI and (minimally) DCA. The present study can provide novel insights into the current management in this field, among 'real-world' patients with CIEDs.

Current guidelines do not address in detail the management of patients with AHREs. Therefore, further studies are needed to explore the role of AHRE for event-risk stratification and decision-making for thromboprophylaxis in AHRE. Some prospective clinical trials are ongoing, which are investigating the benefit of OACs in patients with CIEDs, and will provide useful information on management of patients with AHREs [26, 27].

\section{Limitations}

There are several limitations in the present study. First, this is a single-center, retrospective, and observational study in a hospital-based setting. A relatively small number of patients were included in the present study, which may have reduced the detection power and possibly influenced the validity of some interactions. Second, we collected the clinical data at the time of CIEDs' implantation, while the data at follow-up were not taken into account. As expected, initiation and discontinuation of OAC during follow-up would affect clinical outcomes, and we had limited information on this. Finally, data on quality of anticoagulation such as international normalized ratio or time in therapeutic range were not available in the present study. Notwithstanding the relatively modest size and residual confounding, we found no significant relationship between OAC use and thromboembolism in multivariate Cox regression analysis.

\section{Conclusion}

In conclusion, AHRE was an independent factor associated with increased risk of clinical outcomes. The addition of AHRE to the $\mathrm{CHADS}_{2}$ and $\mathrm{CHA}_{2} \mathrm{DS}_{2}$-VASc scores significantly improved discrimination for thromboembolism or death. 
Funding None directly related to this manuscript.

\section{Compliance with ethical standards}

Conflict of interest No author has a specific conflict of interest in the publication of this study. GYHL: Consultant for Bayer/Janssen, BMS/ Pfizer, Medtronic, Boehringer Ingelheim, Novartis, Verseon and Daiichi-Sankyo; Speaker for Bayer, BMS/Pfizer, Medtronic, Boehringer Ingelheim, and Daiichi-Sankyo. No fees are directly received personally. GB: Speaker for Biotronik, Boehringer Ingelheim, Boston Scientific and Medtronic.

Open Access This article is distributed under the terms of the Creative Commons Attribution 4.0 International License (http://creativeco mmons.org/licenses/by/4.0/), which permits unrestricted use, distribution, and reproduction in any medium, provided you give appropriate credit to the original author(s) and the source, provide a link to the Creative Commons license, and indicate if changes were made.

\section{References}

1. Vermond RA, Geelhoed B, Verweij N, Tieleman RG, Van der Harst P, Hillege HL, Van Gilst WH, Van Gelder IC, Rienstra M (2015) Incidence of atrial fibrillation and relationship with cardiovascular events, heart failure, and mortality: a community-based study from the Netherlands. J Am Coll Cardiol 66:1000-1007

2. Lip GYH (2017) The ABC pathway: an integrated approach to improve AF management. Nat Rev Cardiol 14:627-628

3. Pastori D, Pignatelli P, Menichelli D, Violi F, Lip GYH (2018) Integrated care management of patients with atrial fibrillation and risk of cardiovascular events: the $\mathrm{ABC}$ (atrial fibrillation better care) pathway in the ATHERO-AF study cohort. Mayo Clin Proc 1:1. https://doi.org/10.1016/j.mayocp.2018.10.022

4. Healey JS, Oldgren J, Ezekowitz M et al (2016) Occurrence of death and stroke in patients in 47 countries 1 year after presenting with atrial fibrillation: a cohort study. Lancet 388:1161-1169

5. Gladstone DJ, Bui E, Fang J, Laupacis A, Lindsay MP, Tu JV, Silver FL, Kapral MK (2009) Potentially preventable strokes in high-risk patients with atrial fibrillation who are not adequately anticoagulated. Stroke 40:235-240

6. Savelieva I, Camm AJ (2000) Clinical relevance of silent atrial fibrillation: prevalence, prognosis, quality of life, and management. J Interv Card Electrophysiol 4:369-382

7. Lin HJ, Wolf PA, Benjamin EJ, Belanger AJ, D’Agostino RB (1995) Newly diagnosed atrial fibrillation and acute stroke. The Framingham Study. Stroke 26:1527-1530

8. Mairesse GH, Moran P, Van Gelder IC et al (2017) Screening for atrial fibrillation: a European Heart Rhythm Association (EHRA) consensus document endorsed by the Heart Rhythm Society (HRS), Asia Pacific Heart Rhythm Society (APHRS), and Sociedad Latinoamericana de Estimulacion Cardiaca y Electrofisiologia (SOLAECE). Europace 19:1589-1623

9. Pollak WM, Simmons JD, Interian A Jr, Atapattu SA, Castellanos A, Myerburg RJ, Mitrani RD (2001) Clinical utility of intraatrial pacemaker stored electrograms to diagnose atrial fibrillation and flutter. Pacing Clin Electrophysiol 24:424-429

10. Glotzer TV, Hellkamp AS, Zimmerman J et al (2003) Atrial high rate episodes detected by pacemaker diagnostics predict death and stroke: report of the Atrial Diagnostics Ancillary Study of the MOde Selection Trial (MOST). Circulation 107:1614-1619

11. Glotzer TV, Daoud EG, Wyse DG, Singer DE, Ezekowitz MD, Hilker C, Miller C, Qi D, Ziegler PD (2009) The relationship between daily atrial tachyarrhythmia burden from implantable device diagnostics and stroke risk: the TRENDS study. Circ Arrhythm Electrophysiol 2:474-480

12. Healey JS, Connolly SJ, Gold MR et al (2012) Subclinical atrial fibrillation and the risk of stroke. N Engl J Med 366:120-129

13. Kirchhof P, Benussi S, Kotecha D et al (2016) 2016 ESC Guidelines for the management of atrial fibrillation developed in collaboration with EACTS. Eur Heart J 37:2893-2962

14. DeLong ER, DeLong DM, Clarke-Pearson DL (1988) Comparing the areas under two or more correlated receiver operating characteristic curves: a nonparametric approach. Biometrics 44:837-845

15. Pencina MJ, D'Agostino RB, D’Agostino RB Jr, Vasan RS (2008) Evaluating the added predictive ability of a new marker: from area under the ROC curve to reclassification and beyond. Stat Med 27:157-172 (discussion 207-112)

16. Vickers AJ, Elkin EB (2006) Decision curve analysis: a novel method for evaluating prediction models. Med Decis Making 26:565-574

17. Miyazawa K, Pastori D, Lip GYH (2018) Quantifying time in atrial fibrillation and the need for anticoagulation. Prog Cardiovasc Dis 60:537-541

18. Freedman B, Boriani G, Glotzer TV, Healey JS, Kirchhof P, Potpara TS (2017) Management of atrial high-rate episodes detected by cardiac implanted electronic devices. Nat Rev Cardiol 14:701-714

19. Mahajan R, Perera T, Elliott AD et al (2018) Subclinical devicedetected atrial fibrillation and stroke risk: a systematic review and meta-analysis. Eur Heart J 39:1407-1415

20. Lamas GA, Lee KL, Sweeney MO et al (2002) Ventricular pacing or dual-chamber pacing for sinus-node dysfunction. N Engl J Med 346:1854-1862

21. Sweeney MO, Hellkamp AS, Ellenbogen KA, Greenspon AJ, Freedman RA, Lee KL, Lamas GA, Investigators MOST (2003) Adverse effect of ventricular pacing on heart failure and atrial fibrillation among patients with normal baseline QRS duration in a clinical trial of pacemaker therapy for sinus node dysfunction. Circulation 107:2932-2937

22. Yannopoulos D, Lurie KG, Sakaguchi S, Milstein S, Ermis C, VanHeel L, Benditt DG (2007) Reduced atrial tachyarrhythmia susceptibility after upgrade of conventional implanted pulse generator to cardiac resynchronization therapy in patients with heart failure. J Am Coll Cardiol 50:1246-1251

23. Shanmugam N, Boerdlein A, Proff J, Ong P, Valencia O, Maier SK, Bauer WR, Paul V, Sack S (2012) Detection of atrial highrate events by continuous home monitoring: clinical significance in the heart failure-cardiac resynchronization therapy population. Europace Feb 14:230-237

24. Botto GL, Padeletti L, Santini M et al (2009) Presence and duration of atrial fibrillation detected by continuous monitoring: crucial implications for the risk of thromboembolic events. J Cardiovasc Electrophysiol 20:241-248

25. Boriani G, Botto GL, Padeletti L, Santini M, Capucci A, Gulizia M, Ricci R, Biffi M, De Santo T, Corbucci G, Lip GY, Italian ATRI (2011) Improving stroke risk stratification using the CHADS2 and CHA2DS2-VASc risk scores in patients with paroxysmal atrial fibrillation by continuous arrhythmia burden monitoring. Stroke 42:1768-1770

26. Lopes RD, Alings M, Connolly SJ et al (2017) Rationale and design of the Apixaban for the reduction of thrombo-embolism in patients with device-detected sub-clinical atrial fibrillation (ARTESiA) trial. Am Heart J 189:137-145

27. Kirchhof P, Blank BF, Calvert M, Camm AJ, Chlouverakis G, Diener HC, Goette A, Huening A, Lip GYH, Simantirakis E, Vardas P (2017) Probing oral anticoagulation in patients with atrial high rate episodes: Rationale and design of the Non-vitamin K antagonist Oral anticoagulants in patients with Atrial High rate episodes (NOAH-AFNET 6) trial. Am Heart J 190:12-18 